\title{
Correction to: A comparison of endotracheal tube compensation technique for the measurement of respiratory mechanical impedance at low frequencies
}

\author{
Andrea F. Cruz ${ }^{1,2}(\mathbb{D}) \cdot$ Jacob Herrmann ${ }^{2,3} \mathbb{D} \cdot$ Carlos R. R. Carvalho $^{1}\left(\mathbb{D} \cdot\right.$ David W. Kaczka $^{2,3,4} \mathbb{D}$
}

Published online: 18 January 2022

(c) Springer Nature B.V. 2022

\section{Correction to: \\ Journal of Clinical Monitoring and Computing \\ https://doi.org/10.1007/s10877-021-00788-9}

In the original publication of the article, the typesetters have inadvertently included an additional affiliation to the corresponding author David W. Kaczka. The correct affiliations are as follows. The original article has been corrected.

2. Department of Anesthesia, University of Iowa, Iowa City, IA, USA

3. Roy J. Carver Department of Biomedical Engineering, University of Iowa, Iowa City, IA, USA
4. Department of Radiology, University of Iowa, Iowa City, IA, USA

Publisher's Note Springer Nature remains neutral with regard to jurisdictional claims in published maps and institutional affiliations.

The original article can be found online at https://doi.org/10.1007/ s10877-021-00788-9.

David W. Kaczka

david-kaczka@uiowa.edu

1 Laboratorio de Pneumologia LIM-09, Disciplina de Pneumologia, Heart Institute (Incor), Hospital das Clinicas da Faculdade de Medicina da Universidade de São Paulo, São Paulo, São Paulo, Brazil

2 Department of Anesthesia, University of Iowa, Iowa City, IA, USA

3 Roy J. Carver Department of Biomedical Engineering, University of Iowa, Iowa City, IA, USA

4 Department of Radiology, University of Iowa, Iowa City, IA, USA 